\title{
Metabolomic and Transcriptomic Changes Induced by Potassium Deficiency During Sarocladium oryzae Infection Reveal Insights into Rice Sheath Rot Disease Resistance
}

\author{
Jianglin Zhang ${ }^{1,2}$, Zhifeng Lu, ${ }^{1,2}$, Tao Ren ${ }^{1,2}$, Rihuan Cong ${ }^{1,2}$, Jianwei Lu ${ }^{1,2}$ and Xiaokun Li ${ }^{* *}$
}

\begin{abstract}
Rice sheath rot disease caused by Sarocladium oryzae (S. oryzae) infection is an emerging disease, and infection can cause yield losses of 20-85\%. Adequate potassium (K) application is a feasible strategy for rice tolerance to S. oryzae infection. However, little is known about the metabolic mechanisms regulated by K that allow rice to cope better with S. oryzae infection. The present study performed a comparative metabolome and transcriptome analysis of rice with different $\mathrm{K}$ nutrition statuses before and upon S. oryzae infection. Sarocladium oryzae infection triggered a hydrogen peroxide $\left(\mathrm{H}_{2} \mathrm{O}_{2}\right)$ burst, and $\mathrm{K}$ starvation aggravated the accumulation of $\mathrm{H}_{2} \mathrm{O}_{2}$ in the flag leaf sheath (FLS), which resulted in lipid peroxidation. Likewise, $\mathrm{K}$ deficiency altered the lipid homeostasis of the host plants by hyperaccumulation of 1-alkyl-2-acylglycerophosphoethanolamine. K starvation decreased the content of glycoglycerolipids including monogalactosyldiacyglycerol and digalactosyldoacylglycerol during S. oryzae infection, which destroyed the stability of bilayer membranes. In contrast, sufficient K supply increased antioxidant-related transcript expression (for example, the genes related to glutathione-S-transferase biosynthesis were upregulated), which activated the antioxidant systems. Additionally, upon S. oryzae infection, K starvation amplified the negative impacts of S. oryzae infection on flag leaf photosynthetic potential. These results provide new insight into the role of $\mathrm{K}$ in alleviating S. oryzae infection. Adequate K supply decreased the negative impacts of sheath rot disease on rice growth by alleviating lipid peroxidation and maintaining lipid homeostasis.
\end{abstract}

Keywords: Lipid peroxidation, K deficiency, Sarocladium oryzae infection, Sheath rot, Metabolome and transcriptome

\section{Background}

Rice (Oryza sativa L.) is a widely grown crop worldwide, that plays an important role in food security. Sheath rot (ShR) disease caused by Sarocladium oryzae ((Sawada) W. Gams \& D. Hawksw) is an emerging threat to rice production, and the infection of which causes yield losses of 20-85\% (Sakthivel 2001; Bigirimana et al. 2015). S. oryzae

\footnotetext{
*Correspondence: lixiaokun@mail.hzau.edu.cn

${ }^{1}$ Key Laboratory of Arable Land Conservation (Middle and Lower Reaches of Yangtze River), Ministry of Agriculture and Rural Affairs, People's

Republic of China, Wuhan 430070, China

Full list of author information is available at the end of the article
}

generally invades rice via the flag leaf sheaths (FLSs) during the booting stage, and the typical symptoms are grayish-brown lesions occurring on the FLSs that gradually evolve into rotting of the entire FLSs (Hittalmani et al. 2016). Our previous study demonstrated that rotting of the FLSs inhibited the exportation of non-structural carbohydrates from the stems and FLSs to the grains during the grain-filling process, which profoundly decreased the seed-setting rate and final grain yield (Zhang et al. 2019a). Therefore, the suppression of rotting is crucial for enhancing rice tolerance to $S$. oryzae infection.

The tolerance capacity of host plants is often associated with cell-tissue metabolic processes, such as amino 
acid and lipid metabolism, which directly take part in plant-pathogen interactions (Fagard et al. 2014; Rojas et al. 2014). Lipids provide the basic component of cell membranes, which act as the first line of defense against pathogen invasion (Adigun et al. 2021). To attack plants, fungal pathogens generally secrete toxins that target plant lipid metabolism and trigger an oxidative burst. A previous study reported that infection by $S$. oryzae results in the secretion of cerulenin, which may disturb fatty acid biosynthesis and lipid metabolism (Bigirimana et al. 2015). As an immune response, an oxidative burst protects plants against pathogenic infection; however, the consistent accumulation of hydrogen peroxide $\left(\mathrm{H}_{2} \mathrm{O}_{2}\right)$ induces lipid peroxidation, which, when it exceeds a certain threshold level, may contribute to membrane damage and cell death (Triantaphylidès et al. 2008; Nita and Grzybowski 2016). The tolerance that plants usually display against infection by a fungal pathogen occurs by maintaining the stability of cellular membranes and regulating lipid homeostasis (Raffaele et al. 2009). Examples include regulating the contents of monogalactosyldiacylglycerol (MGDG) and digalactosyldiacylglycerol (DGDG), which are essential for maintaining the integrity and stability of cellular membranes in response to abiotic and biotic stresses (Perlikowski et al. 2016; Zhang et al. 2019b). S. oryzae infection may also induce oxidative stress, further resulting in cell death (Bigirimana et al. 2015). However, the alterations in the lipid metabolic processes of the host plant that cope with $S$. oryzae infection remain unknown. Considering that ShR encloses young panicles, it is difficult to control this disease by using chemical fungicides. Thus, regulating the host plant's metabolism to improve its tolerance ability is an important strategy against $S$. oryzae infection.

Potassium (K) is the most abundant of the univalent cations in rice, and its application profoundly improves plant tolerance to infection by most fungal pathogens (Amtmann et al. 2008). Despite the fact that $\mathrm{K}$ is not metabolized in the host plant, the activation of enzymes, maintenance of cell membrane stability, and promotion of antioxidant biosynthesis are modulated by cytoplasmic $\mathrm{K}^{+}$concentrations, and all these processes are essential for plant-pathogen interactions (Marschner 2012). Notable, plant $\mathrm{K}$ deficiency generally induces higher NADPH oxidase activity, which accelerates the accumulation of $\mathrm{H}_{2} \mathrm{O}_{2}$ in K-starved plants (Shin and Schachtman 2004). As mentioned previously, excessive $\mathrm{H}_{2} \mathrm{O}_{2}$ accumulation induces electrolyte leakage and causes cell death (Demidchik et al. 2014). Therefore, long-term $K$ deficiency in rice may aggravate oxidative stress under biotic stress conditions, which manifests in K-starved plants as hyperaccumulation of $\mathrm{H}_{2} \mathrm{O}_{2}$ and malondialdehyde (MDA); this process causes membrane lipid peroxidation $(\mathrm{Hu}$ et al. 2016). Our previous study demonstrated that $S$. oryzae destroyed the metabolic balance and that $\mathrm{K}$ deficiency aggravated electrolyte leakage during the infection process (Zhang et al. 2019a, 2020). However, the metabolic mechanisms of host plants regulated by $\mathrm{K}$ nutrition to cope with S. oryzae infection are still imperfectly known.

Previous studies demonstrated that K deficiency promoted the biosynthesis of oxylipin (Troufflard et al. 2010; Armengaud et al. 2010). Furthermore, S. oryzae infection profoundly altered the hormone levels of the host plant, such as the content of abscisic and jasmonic acids (Peeters et al. 2020). Notably, lipid metabolism provides a substrate for the biosynthesis of jasmonic acid and oxylipids (Conconi et al. 1996; Torres-Franklin et al. 2009). Thus, exploring the whole metabolic profile of host plants at different $\mathrm{K}$ levels during $S$. oryzae infection could advance our understanding of the important role of $\mathrm{K}$ nutrition in rice against ShR disease. In this study, comparative metabolome and transcriptome analyses were performed to investigate the alterations in metabolites and transcriptome profiles between K-starved and K-sufficient rice during $S$. ory$z a e$ infection. The main objectives of this study were to select the predominant metabolic pathways regulated by K during S. oryzae infection and to explore the metabolic mechanisms of rice as regulated by $\mathrm{K}$ to cope with sheath rotting. Our findings provide comprehensive information on the metabolome, transcriptome, and ionome dynamics regulated by $\mathrm{K}$ during the infection process.

\section{Results \\ Low-K Rice Leaf Sheaths are Hypersensitive to S. oryzae Infection}

The typical symptoms of ShR disease were observed in the FLSs upon S. oryzae inoculation. The minimum fluorescence $\left(\mathrm{F}_{\mathrm{o}}\right)$ was highest, while the maximum quantum efficiency of PSII photochemistry $\left(\mathrm{F}_{\mathrm{v}} / \mathrm{F}_{\mathrm{m}}\right)$ was lowest in the $-\mathrm{K}+\mathrm{I}$ treatment, which meant that $\mathrm{K}$-starved rice was more sensitive to $S$. oryzae infection (Fig. 1a). The lesion length in the K-starved rice was significantly longer than that in the K-sufficient rice (Fig. 1b). Comparative metabolome and transcriptome analyses were used to examine the mechanisms underlying the further aggravation of $S$. oryzae infection by K deficiency. Before inoculation, 246 genes were significantly upregulated in the K-starved rice, and a fewer number of genes were downregulated (Fig. 1c). The K-starved rice differed significantly from the K-sufficient rice at the metabolic and transcription levels (Fig. 1d). Upon S. oryzae infection, principal component analysis clearly separated the infected and uninfected treatments by their metabolite profiles, transcripts, and ion contents. $\mathrm{K}$ starvation enhanced the differences in metabolites, transcripts, and ion contents between $S$. oryzae infected and uninfected FLSs (Fig. 1e). 


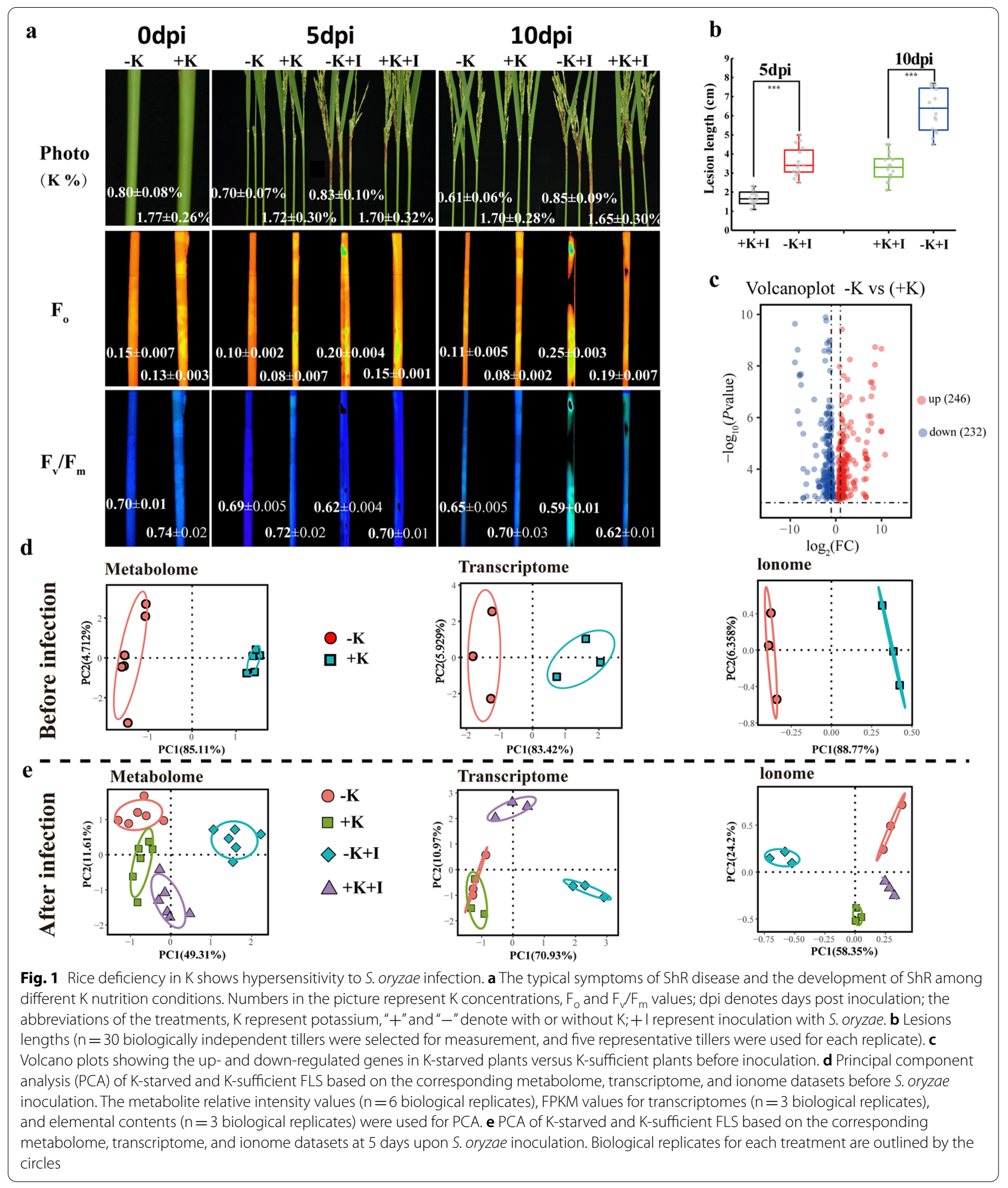


K Deficiency Induces Larger Alterations in the Predominant Metabolic Pathways Under S. oryzae Infection

To assess how K deficiency aggravates $S$. oryzae infection by altering metabolic processes in the FLSs, we determined the quantitative profiles of 147 metabolites in the FLSs using nontargeted LC-MS/MS. Most of the metabolites related to amino acids, carbohydrates, lipids, nucleotides, secondary metabolism, and hormone metabolism were upregulated upon $S$. oryzae infection (Fig. 2).

We further organized all of the metabolites using Cytoscape software (Shannon et al. 2003). These compounds

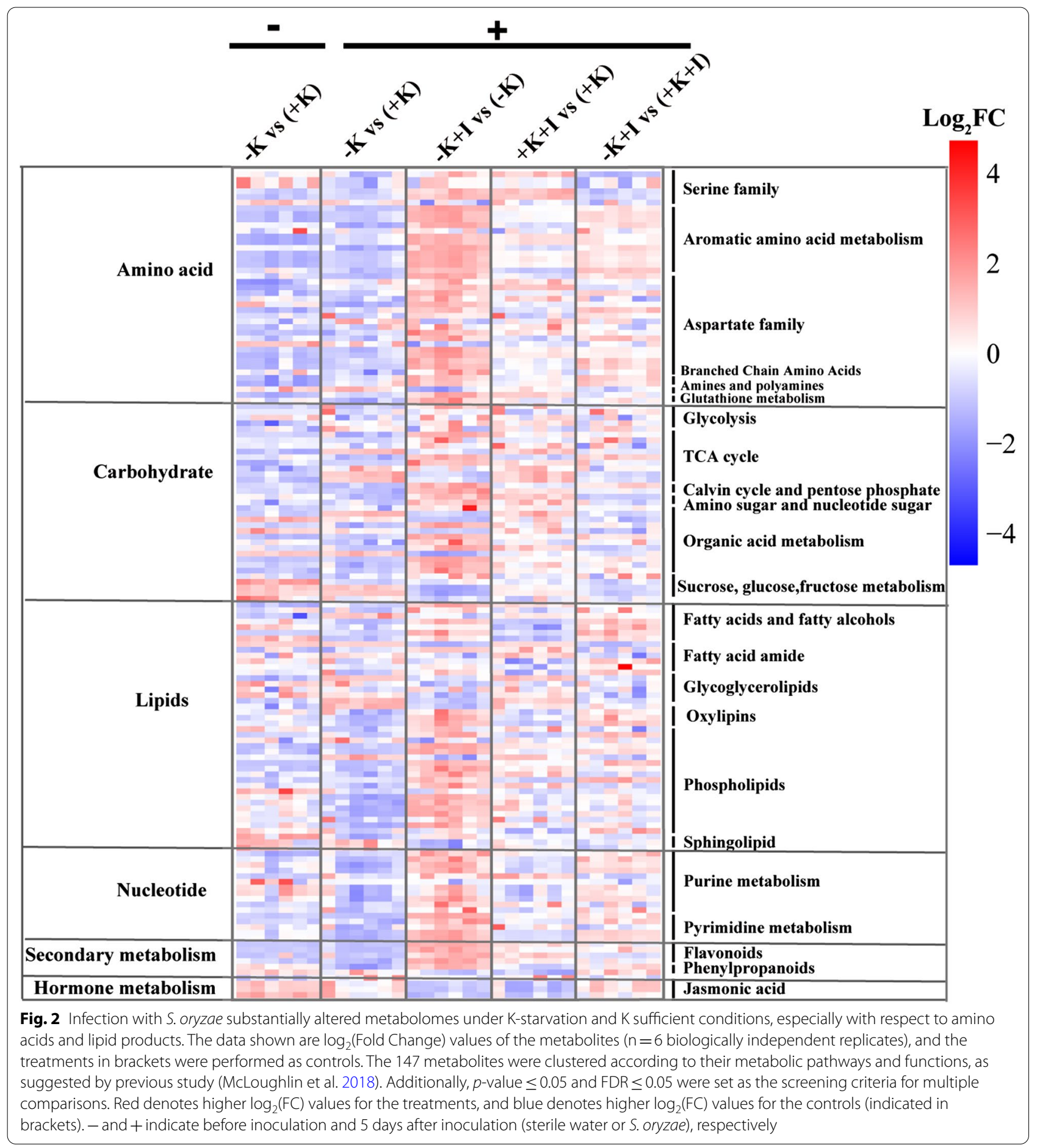




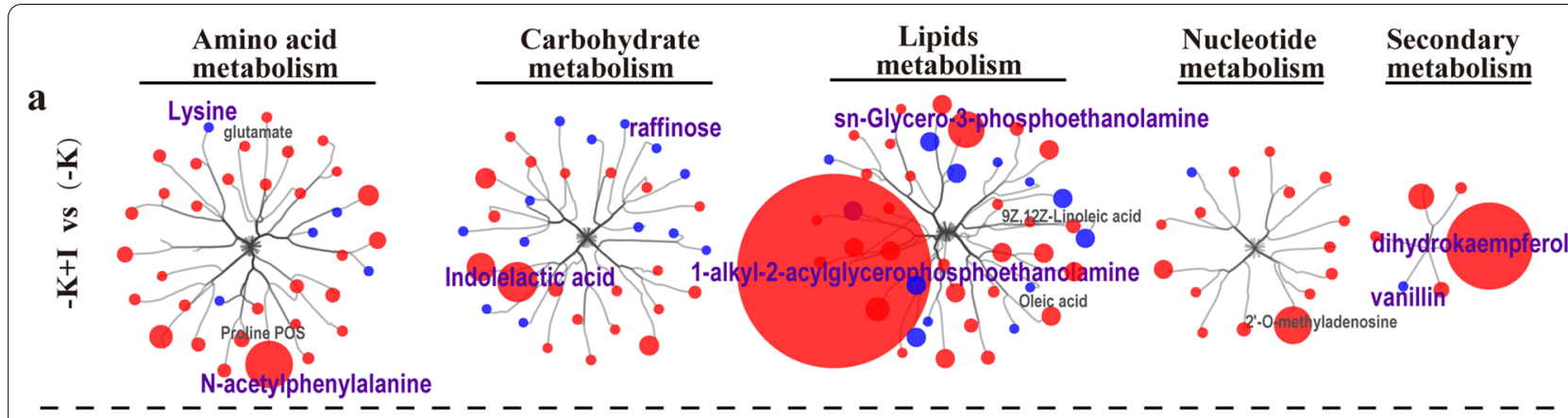

b
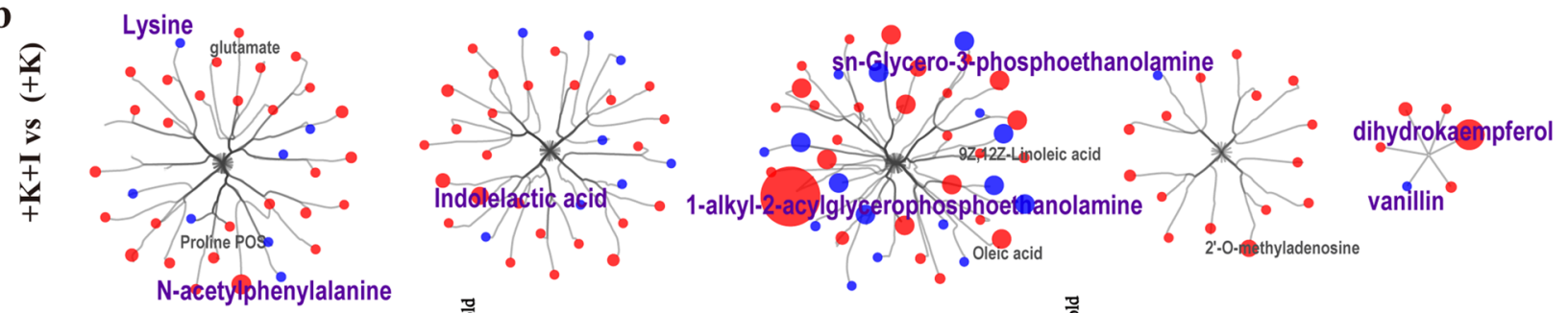

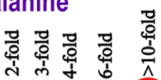

Up-regulated by infection

c
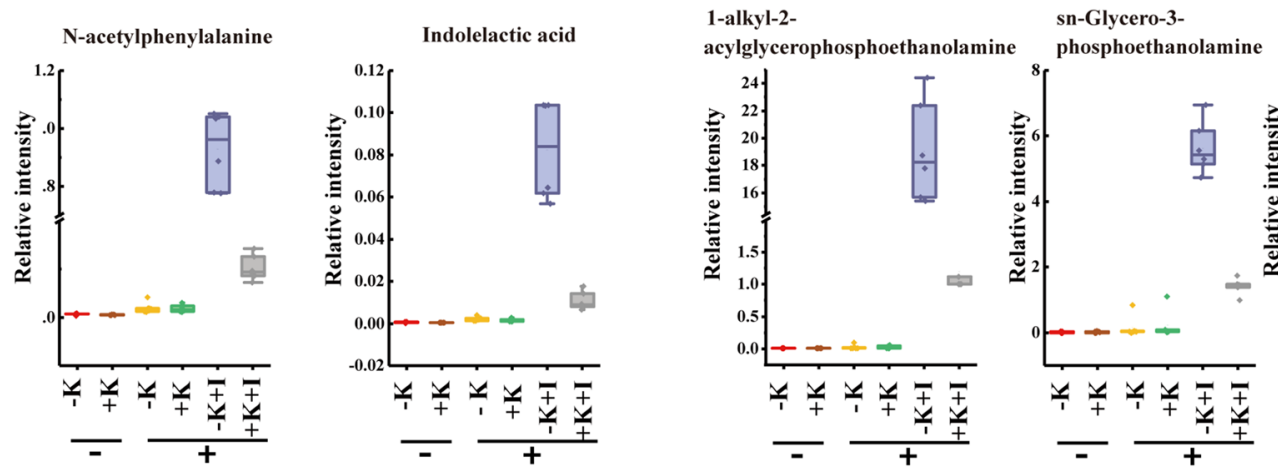

dihydrokaempferol

Fig. 3 Survey of metabolic processes indicates that lipid metabolism is severely affected by S. oryzae infection. a Effects of S. oryzae infection on the metabolic profile of K-starved plants (at 5 days after inoculation). The metabolites were clustered using Cytoscape and the sizes of the circles denote the FC values ( $n=6$ biological replicates). $\mathbf{b}$ Effects of S. oryzae infection on the metabolic profile of K-sufficient plants. Blue indicates higher FC values for uninfected plants, and red denotes higher FC values for diseased plants; the treatments in brackets were performed as controls. c Examples of compounds substantially altered by S. oryzae infection. The center line in each box plot represents the mean relative intensity ( $\mathrm{n}=6$ )

were clustered according to their function. The most obvious alterations were found in amino-acid metabolism and lipid metabolism (Fig. 3a, b). Lipid-breakdown products accumulated significantly in K-starved FLSs upon S. oryzae infection. For instance, the relative content of 1-alkyl-2-acylglycerophosphoethanolamine, a phospholipid, increased 18-fold in K-starved rice under S. oryzae infection compared to the corresponding value in the $+\mathrm{K}+\mathrm{I}$ treatment (Fig. 3c). Other obvious changes in diseased FLSs were observed among compounds related to carbohydrate metabolism and secondary metabolism. For example, vanillin (belonging to secondary metabolism), which is associated with phenylpropanoid biosynthesis, was downregulated in the diseased rice
(Fig. 3). The content of indoleacetic acid (IAA) in the diseased tissues was up-regulated, which consistent with a previous study reported that $S$. oryzae infection triggered strong IAA responses and IAA levels positively correlated with the lesion area (Peeters et al. 2020). Dihydrokaempferol is a derivative of flavonoids, the upregulation of which indicates that anthocyanin metabolism is activated.

\section{Metabolic and Transcriptomic Analyses Show That Membrane Lipid Peroxidation is Highly Induced by $\mathrm{K}$ Deficiency Under S. oryzae Infection}

Lysine and raffinose, which function as indicators of host plant tolerance to oxidative stress (Nishizawa et al. 2008), 
were down-regulated upon S. oryzae infection (Fig. 3). We combined metabolome analysis and Illumina-based RNA sequencing to further analyze alterations in the predominant metabolic pathways and gene expression profiles. All metabolites were annotated via the Kyoto Encyclopedia of Genes and Genomes (KEGG) analysis, top-20 significantly enriched pathways were selected based on $p$-values and impact factors (Fig. 4). The aminoacyl-tRNA biosynthesis pathway was significantly altered in the diseased FLSs, irrespective of $\mathrm{K}$ nutrition status (Fig. 4a, b). Before S. oryzae infection, K deficiency profoundly influenced the process of phenylpropanoid biosynthesis (Fig. 4c). However, S. oryzae infection increased the differences in linoleic acid metabolism between K-starved and K-sufficient rice (Fig. 4d).

FPKM values were used to identify the differentially expressed genes (DEGs). Absolute values of $\log _{2}(\mathrm{FC})>1.5, p$-value $<0.01$, and $\mathrm{FDR}<0.05$ were used to define DEGs. More DEGs were up-regulated in the K-deficient plants (1726 DEGs) than

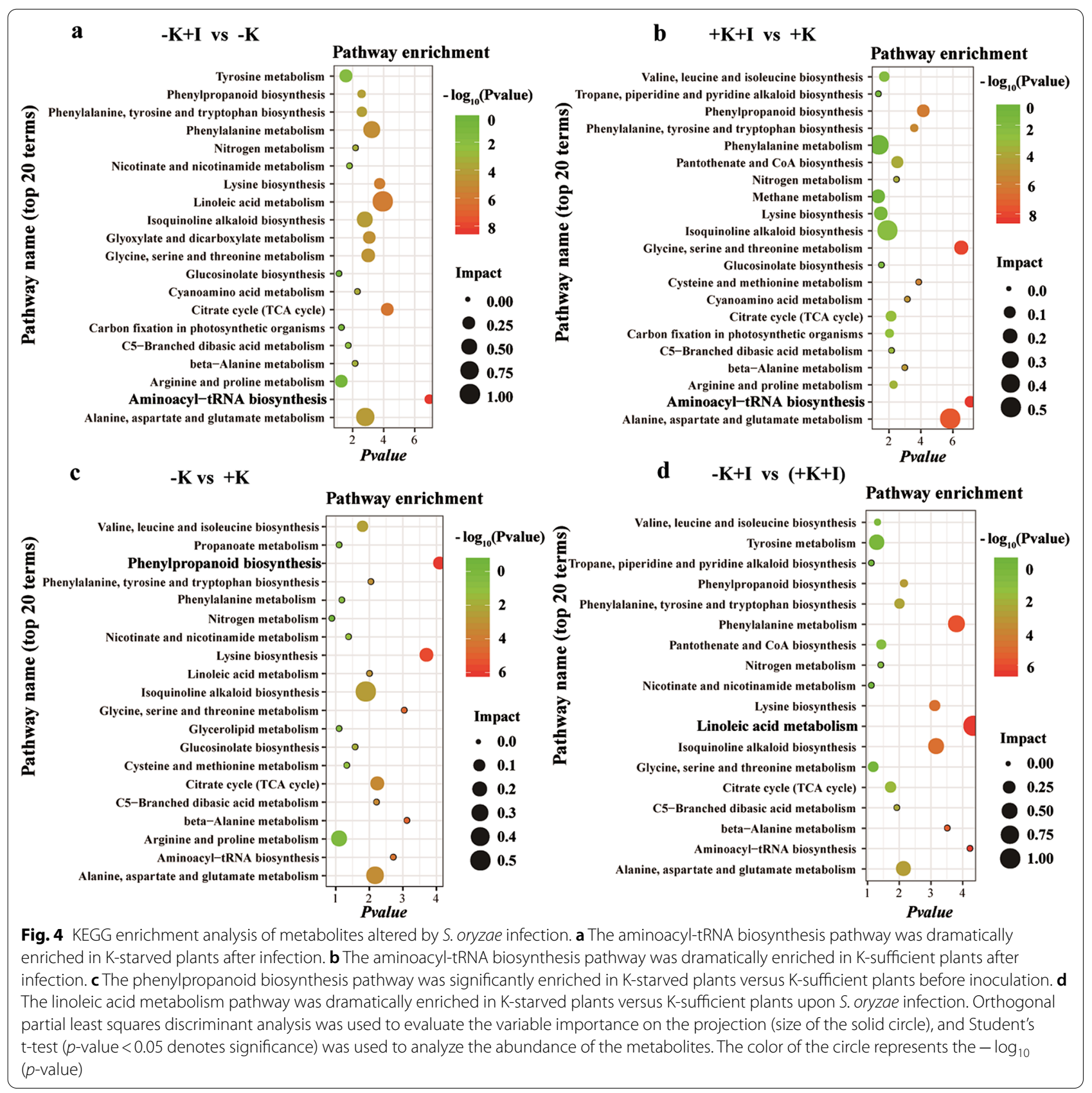



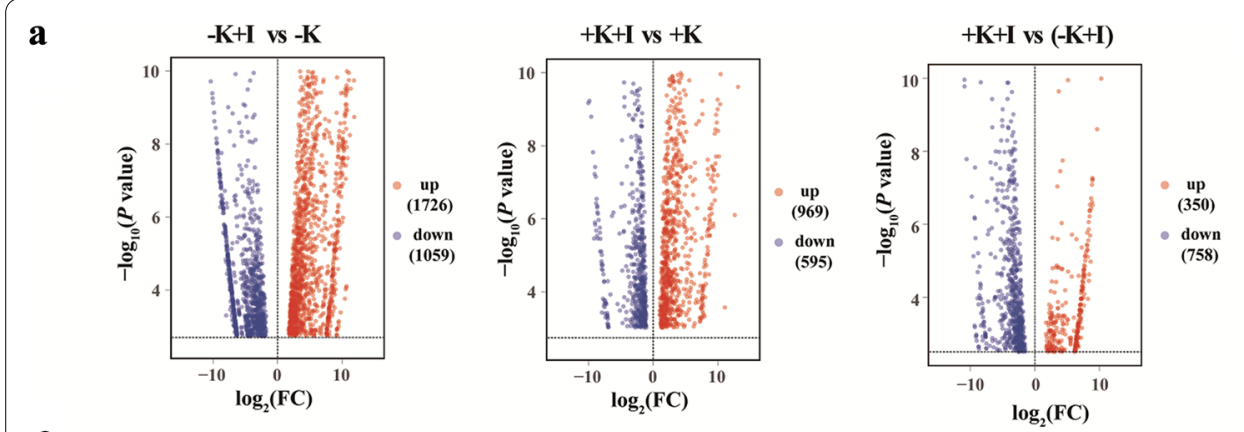

b


d
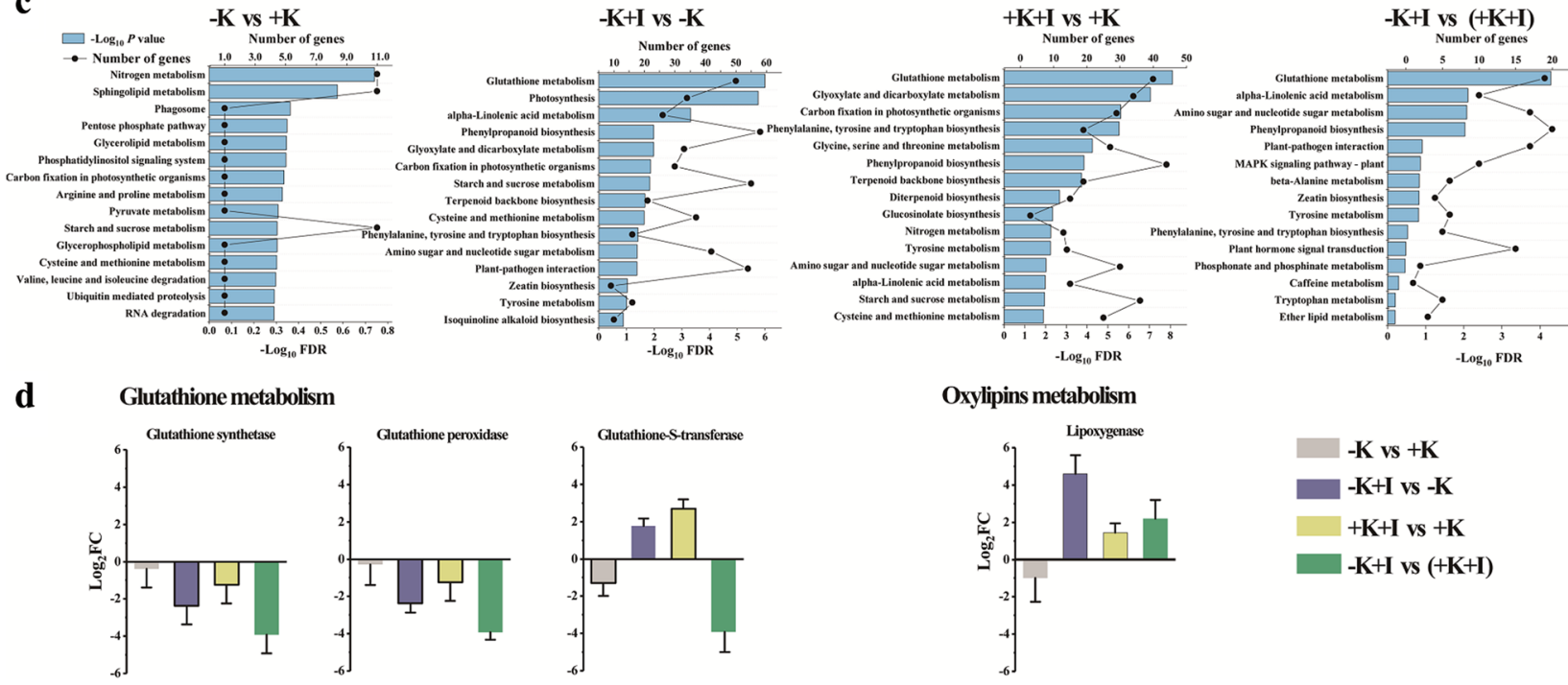

Fig. 5 The rice transcriptome is significantly altered by S. oryzae infection under both K-starvation and K-rich conditions. a Volcano plots showing the up- and down-regulated genes in K-starved and K-sufficient rice upon S. oryzae infection. $\mathbf{b}$ Numbers of overlapped transcripts in diseased and healthy plants under K-rich and K-starvation conditions. c KEGG enrichment of DEGs between K-starved and K-sufficient rice under both infected and non-infected conditions. The glutathione and lipid metabolism pathways were obviously enriched in K-starved rice under S. oryzae infection. $\mathbf{d}$ $\log _{2}(\mathrm{FC})$ values of representative transcripts from specific KEGG pathways (see $\left.\mathbf{c}\right)$. Treatments in brackets were performed as the controls

in the K-sufficient plants (969 DEGs) during infection (Fig. 5a). Independent of the $\mathrm{K}$ nutrition status of rice, 290 DEGs were found in the infected FLSs (Fig. 5b). Based on Gene Ontology (GO) annotation analyses, the DEGs were predominantly related to the membrane and membrane parts (Additional file 1: Fig. S1). As a response to oxidative stress and the damage to membrane parts caused by $S$. oryzae infection, the glutathione metabolism pathway was significantly enriched, as shown by KEGG pathway enrichment analysis of the DEGs (Fig. 5c). Additionally, upon $S$. oryzae infection, the representative transcripts for glutathione metabolism, such as the relative expressions of glutathione synthetase, glutathione peroxidase, and Glutathione-S-transferase, were up-regulated in K-sufficient plants compared the K-starved plants (Fig. 5d). By contrast, transcripts related to oxylipin metabolism, such as lipoxygenase were up-regulated in K-starved rice compared with
K-sufficient rice upon S. oryzae infection (Fig. 5d). Most of the genes related to lipid metabolism, especially genes related to phospholipase $\mathrm{D}$, were downregulated in the K-deficient FLS after infection (Additional file 1: Table S2).

\section{K deficiency Amplified the Decrease in Galactolipids Content and Photosynthetic Capacity During S. oryzae Infection}

MDA is an indicator of membrane peroxidation, and $\mathrm{K}$ deficiency aggravated the accumulation of MDA in the FLSs during the infection process (Fig. 6a). Furthermore, $S$. oryzae infection can stimulate a burst of active oxygen. The content of $\mathrm{H}_{2} \mathrm{O}_{2}$ was up-regulated in K-sufficient plants within a short time from infection, followed a gradual stabilization. Conversely, K deficiency consistently induced the accumulation of $\mathrm{H}_{2} \mathrm{O}_{2}$ in the FLSs during infection. Likewise, $\mathrm{K}$ deficiency decreased the superoxide dismutase (SOD) 


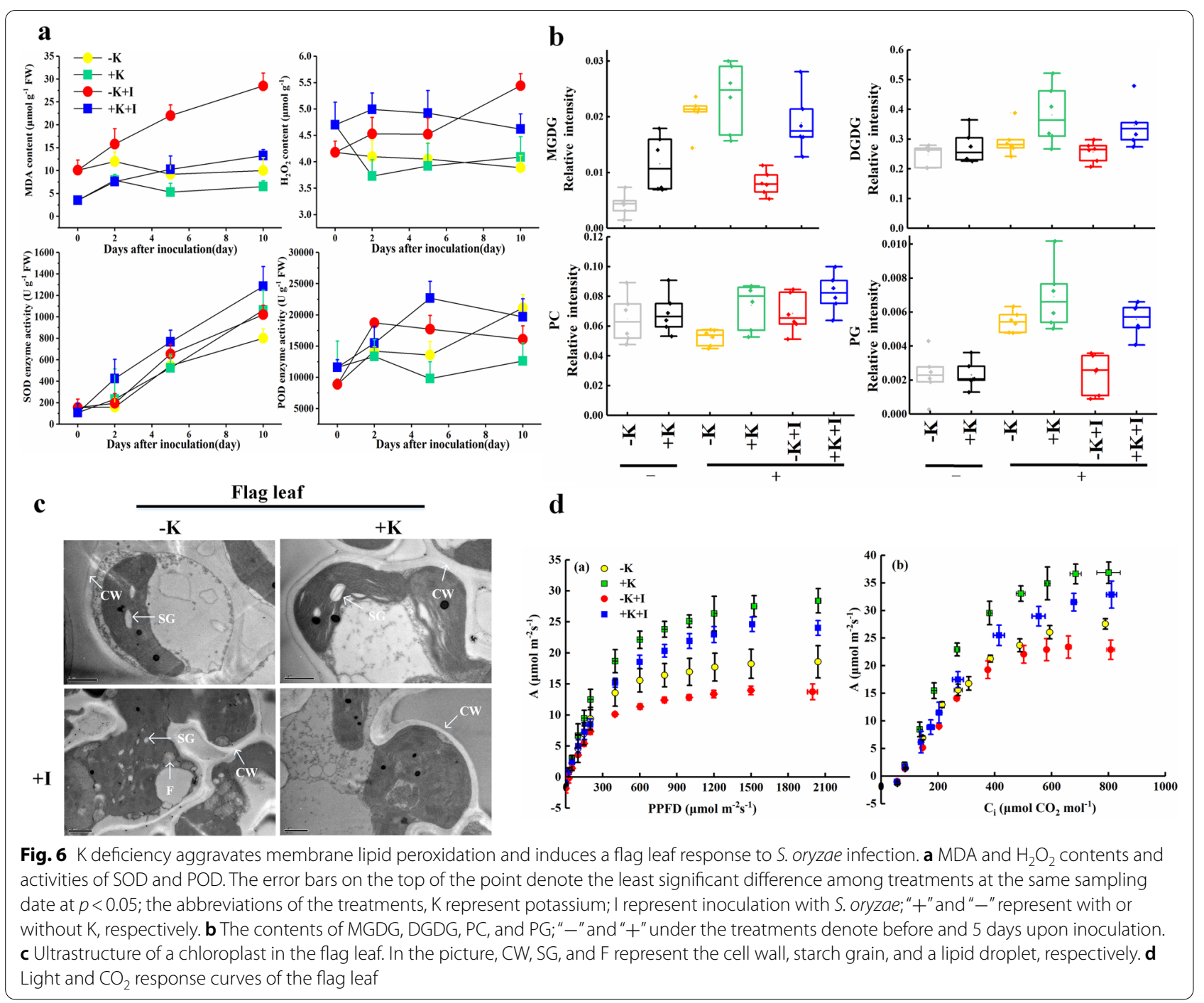

and peroxidase (POD) activities during infection (Fig. 6a). Additionally, upon S. oryzae infection, K deficiency decreased the contents of MGDG, DGDG, phosphatidylcholine (PC) and phosphatidylglycerol (PG) by averages of $57.2 \%, 25.6 \%, 25.8 \%$, and $57.6 \%$ respectively, in comparison with the $+\mathrm{K}+\mathrm{I}$ treatment (Fig. 6b). Most phospholipid species, including PC and PG, were down-regulated in K-starved rice upon S. oryzae infection (Additional file 1: Table S3). As a systematic response of rice to $S$. oryzae infection, K deficiency altered the shape of the chloroplasts in the flag leaf (Additional file 1: Table S4). Likewise, lipid droplets occurred in K-starved plants during infection (Fig. 6c). The light and $\mathrm{CO}_{2}$ response curves showed that the photosynthetic potential of the flag leaf was inhibited by S. oryzae infection (Fig. 6d). K deficiency significantly decreased the net photosynthetic rate
$(A)$, maximum electron transport rate $\left(J_{\max }\right)$, maximum carboxylation rate $\left(V_{\text {cmax }}\right)$, effective quantum efficiency of PSII ( $\phi$ PSII), and $\mathrm{F}_{\mathrm{v}} / \mathrm{F}_{\mathrm{m}}$ during $S$. oryzae infection in comparison with those in K-sufficient plants (Additional file 1: Table S5).

\section{Discussion}

$\mathrm{K}$ helps to improve crop tolerance to biotic stresses through different processes, such as by regulating the morphology, altering the metabolic profiles, and regulating ion homeostasis of the host plant (Amtmann et al. 2008; Shi et al. 2018). ShR disease caused by S. ory$z a e$ infection is a newly emerging fungal disease in rice with specific infection strategies (Bigirimana et al. 2015). However, the metabolic processes of the host plant regulated by K that allow it to cope with $S$. oryzae infection have seldom been revealed. The present study focused on 


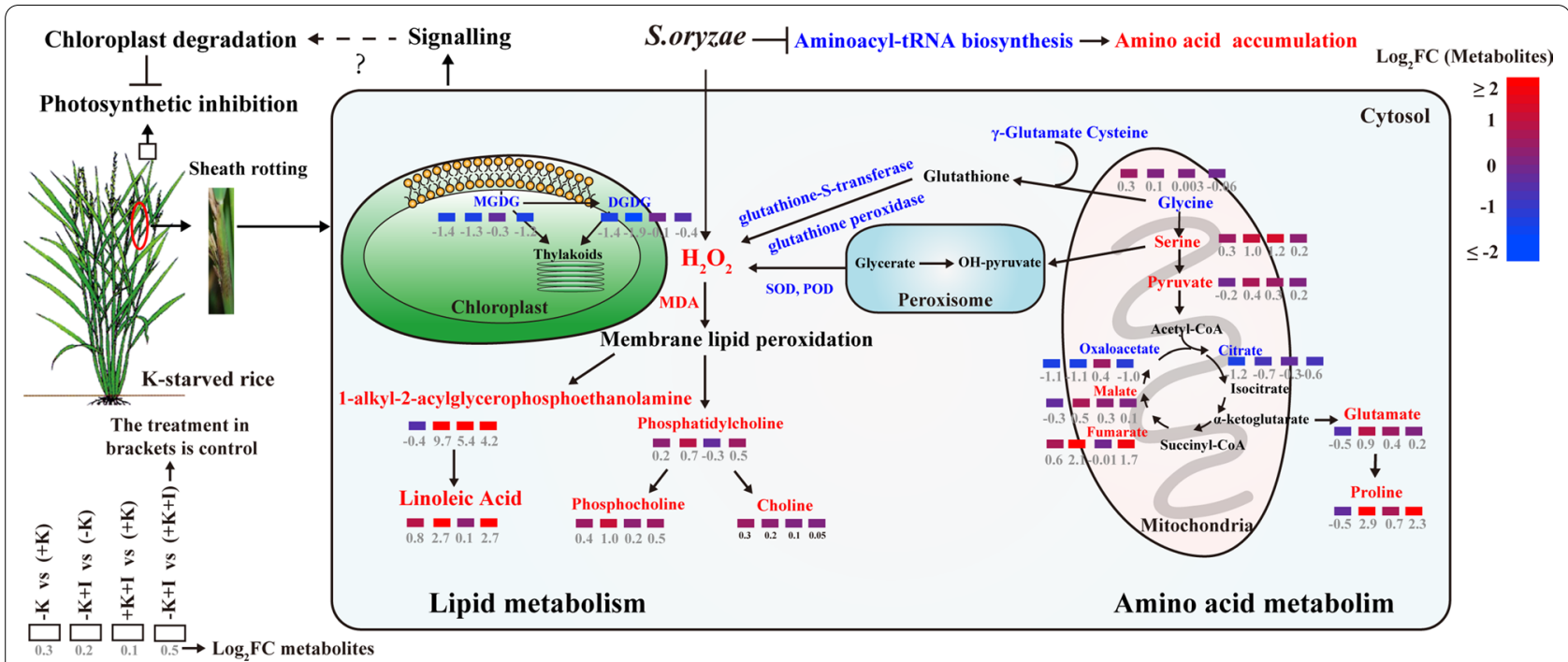

Fig. 7 Summary model of the metabolic processes underlying the response of K-starved rice to S. oryzae infection. First, S. oryzae invades rice from FLSs and causes $\mathrm{H}_{2} \mathrm{O}_{2}$ accumulation, which results in severe oxidative stress. However, MGDG and DGDG contents, which are essential for maintaining membrane integrity and stability, were profoundly decreased in the K-starved FLSs. Likewise, K deficiency also decreases the synthesis of the antioxidant glutathione and the activities of SOD and POD. The consistent accumulation of $\mathrm{H}_{2} \mathrm{O}_{2}$ in $\mathrm{K}$-starved rice induces lipid peroxidation, which accelerates 1-alkyl-2-acylglycerophosphoethanolamine and linoleic acid accumulation in K-starved FLSs. Second, S. oryzae infection impacts the biosynthesis of aminoacyl-tRNA, which results in amino acid accumulation in FLSs. Proline is upregulated and citrate is downregulated in $\mathrm{K}$-starved rice, implying that energy metabolism and osmotic balance are disrupted by S. oryzae infection. As a systematic response, S. oryzae infection also induces the degradation of chloroplasts in flag leaves, which down regulates the photosynthetic rate of K-starved rice. Overall, alterations in these series of metabolic processes in K-starved rice promote S. oryzae development and sheath rotting. Note: FC in metabolite abundance for the treatments versus controls was calculated. The $\log _{2}(F C)$ is indicated by the shade of red or blue according to the color scale, and the numbers below the rectangle indicate the corresponding $\log _{2}(\mathrm{FC})$ values. The metabolites or physiological parameters in red/blue represent up/down regulation in $-\mathrm{K}+\mathrm{I}$-treated plants versus $+\mathrm{K}+\mathrm{I}$-treated plants. The symbol "?" means that the mechanism is not yet clear

the infection site (FLSs). We found that $S$. oryzae invades rice from FLSs and causes an $\mathrm{H}_{2} \mathrm{O}_{2}$ burst, which causes oxidative stress. However, $\mathrm{K}$ deficiency aggravated oxidative stresses and altered amino acid and lipid metabolism (Fig. 7). K starvation accelerated 1-alkyl-2-acylglycerophosphoethanolamine and linoleic acid hyperaccumulation, which further altered the lipid homeostasis of the host plant. In contrast, an adequate $\mathrm{K}$ supply decreased the accumulation of amino acid and lipid metabolism products by alleviating lipid peroxidation (Fig. 7). In the following, we discuss the effects of lipid peroxidation modulated by $\mathrm{K}$ on lipid metabolism and sheath rotting, followed by discussing the systematic responses of rice to S. oryzae infection under different K nutrition statuses.

\section{K Deficiency Alters Lipid Metabolism by Amplifying Lipid Peroxidation During S. oryzae Infection}

Lipid metabolism involves the cell membrane process, which is crucial for rice-microbe interactions, and the peroxidation or degradation of the membrane contributes to cell death (Raffaele et al. 2009; Zoeller et al. 2012; Gao et al. 2017). However, in the present study, most glycerolipids including MGDG and DGDG, were downregulated upon S. oryzae infection (Fig. 2). In contrast, 1-alkyl-2-acylglycerophosphoethanolamine was hyperaccumulated in K-starved FLSs, altering the lipid homeostasis of the host plant. Fungal pathogen infection generally induces lipid peroxidation by causing oxidative stress (Zoeller et al. 2012). Invasion of cell tissues by $S$. oryzae can trigger a hypersensitivity response (HR) in the host plant. The characteristics of HR are NADPH oxidase initiation of an oxidative burst and production of $\mathrm{O}_{2}{ }^{-}$at the cellular membrane (Torres et al. 2005). As the major source of reactive oxygen species (ROS) in the host plant, NADPH oxidase is found at relatively higher contents in K-starved plants (Shin and Schachtman 2004). Therefore, $\mathrm{K}$ starvation amplified oxidative stress in $S$. oryzae-infected tissues (Figs. 5c, 6a). Lipid peroxidation caused by excessive accumulation of ROS may result in cell membrane damage (Zoeller et al. 2012), which is consistent with our result that the MDA content (an indicator of membrane peroxidation) in K-starved rice was significantly higher than that in K-sufficient rice (Fig. 6a). Using trypan blue staining, we also visualized the ShR tissues, and the results indicated that the rate of cell death was much higher in the K-starved FLSs (Additional file 1: 
Fig. S2). Pathogen infection that induces lipid peroxidation exceeding a certain threshold level can contribute to membrane damage and cell death (Triantaphylidès et al. 2008). In this study, $K$ deficiency significantly amplified lipid peroxidation and sheath rotting during S. oryzae infection. In contrast, the presence of sufficient $\mathrm{K}$ increased the biosynthesis of antioxidants (e.g., raffinose and glutathione) (Figs. 5c, d, 7), implying that a protective response is activated in K-sufficient rice to ameliorate the oxidative damage. This is consistent with a previous study indicating that sufficient $\mathrm{K}$ application significantly alleviates oxidative stress ( $\mathrm{Hu}$ et al. 2016). In this study, glutathione transferase was significantly upregulated in K-sufficient rice (Fig. 5d), which mediated the stress tolerance of the host plant under biotic stress (Nianiou-Obeidat et al. 2017).

Additionally, lipid metabolism regulates plant-pathogen interactions predominantly through three strategies: the production of oxylipin or jasmonic acid (JA) in the lipoxygenase pathway, the reconstruction of membrane lipid defense signals in the unsaturated fatty acid pathway, and the synthesis of long-chain fatty acids (Raffaele et al. 2009). In the present study, glycoglycerolipids (MGDG and DGDG) and most phospholipids (PC, $\mathrm{PG}$, and phosphatidylethanolamine) were downregulated in K-starved rice upon S. oryzae infection (Fig. 6b, Additional file 1: Table S3). This result is similar to that of a previous study reporting that Magnaporthe oryzae (fungal pathogen) infection significantly decreased the MGDG and DGDG contents (Zhou et al. 2008). However, some contrary results indicated that MGDG and DGDG contents in Arabidopsis were significantly upregulated by Pseudomonas syringae infection in a short time $(24 \mathrm{~h})$ (Zoeller et al. 2012). In our study, the downregulation of MGDG and DGDG was a strategy of S. oryzae for successful invasion of cell tissues. A previous study reported that lower levels of MGDG and DGDG can suppress immune responses in rice (Gao et al. 2017). In this study, we did not investigate the direct effects of MGDG and DGDG on $S$. oryzae colonization. However, a previous study demonstrated that a higher DGDG/MGDG ratio is an accommodative strategy for sustaining bilayer membranes under stress conditions (Perlikowski et al. 2016). Our results showed that the DGDG/MGDG ratio was significantly lower in K-starved rice (0.17) than in K-sufficient rice (0.31) during $S$. oryzae infection (Fig. 6b), which is in agreement with our hypothesis that $\mathrm{K}$ deficiency aggravates the degradation of the bilayer membrane during S. oryzae infection. Likewise, genes related to phospholipase $\mathrm{D}$ were downregulated by $\mathrm{K}$ deficiency during the infection process (Additional file 1: Table S2). The downregulation of phospholipase D activity might have been associated with remodeling or degradation of the lipid membrane (Adigun et al. 2021). Sufficient K supply significantly decreased the transcript expression of lipoxygenase (Fig. 5d), which might have suppressed the conversion of glycerolipids into oxylipins during $S$. ory$z a e$ infection. However, the content of JA in the FLSs did not increase (Fig. 2), which means that the conversion of linolenic acids into JA might have been suppressed. Likewise, $S$. oryzae infection produces cerulenin and helvolic acid, which destroy fatty acid metabolism and result in chlorosis symptoms, and the degradation of chloroplasts produces linoleic acid or linolenic acids in cell tissues as a response to environmental stress conditions (Conconi et al. 1996; Torres-Franklin et al. 2009; Bigirimana et al. 2015). These findings outline the possible reasons why linolenic acids and 1-alkyl-2-acylglycerophosphoethanolamine hyperaccumulated in K-starved FLSs during S. oryzae infection (Figs. 3a, 4d, 7). Changes in oleic acid levels are closely correlated with JA- triggered plant defense responses (Ruan et al. 2019). JA is initially synthesized from linolenic and linoleic acid. Thus, the hyperaccumulation of linoleic acid may suppress the JA level of the host plant, which is in agreement with a previous report that ShR infection slightly decreased the JA content of the host plant (Peeters et al. 2020). In the present study, we only investigated the effects of K starvation on lipid metabolism and lipid peroxidation during S. ory$z a e$ infection; however, other nutrient deficiencies might also induce membrane peroxidation, and whether other nutrient deficiencies could also promote ShR development needs further study.

\section{K Deficiency Alters Amino-Acid Metabolism and Photosynthetic Potential During S. oryzae Infection}

In the present study, S. oryzae infection destroyed the integrity and stability of cell membranes. Infection with S. oryzae results in the secretion of phytotoxin into cells (Hittalmani et al. 2016), which may suppress the biosynthesis of aminoacyl-tRNA (Fig. 4a, b). Because aminoacyl-tRNA is used for amino acid transfer into the ribosome for protein synthesis (Duchêne et al. 2005; Sheppard et al. 2008), its suppression resulted in amino acid hyperaccumulation in the FLSs (Fig. 2). K deficiency aggravated the accumulation of amino acids in the FLSs and further altered the osmotic characteristics and mineral nutrient homeostasis of the host cells (Additional file 1: Fig. S3). The accumulation of amino acids and organic acids in the FLSs decreased the $\mathrm{pH}$ of the cell microenvironment, and the consistent accumulation of $\mathrm{H}_{2} \mathrm{O}_{2}$ at the infection site(s) supported this result (Fig. 6a). As $\mathrm{K}^{+}$is essential for chloroplast osmoregulation and integrity regulation, an increasing $\mathrm{K}^{+}$concentration regulates the $\mathrm{pH}$ of the cell microenvironment by offsetting the $\mathrm{H}^{+}$concentration (Kunz et al. 2014). 
Maintaining a proper $\mathrm{pH}$ in the cell microenvironment is a feasible strategy for the host plant against fungal pathogen infection (Robison et al. 2018). Thus, in the present study, the K-starved rice exhibited an increasing trend of $\mathrm{K}^{+}$at the infection site (Additional file 1: Fig. $\mathrm{S} 3)$. However, the increased $\mathrm{K}^{+}$content in the $\mathrm{K}$-starved FLSs was at the expense of decreasing $\mathrm{K}^{+}$content at uninfected site(s), such as stems (Additional file 1: Fig. S4). In this study, most of the elemental contents were diminished slightly with an extension of the infection time, which was due mainly to the exportation of elements from the FLSs to the grains due to grain-filling. However, $\mathrm{K}$ deficiency delayed the exportation of most elements from the infected FLSs (Additional file 1: Fig. S3), possibly due to the destroyed cell membranes inhibiting the phloem-loading process at the infection site(s) (Chen 2014). Additionally, as a systematic response, fungal pathogen infection induced the HR process, which reduced the photosynthetic rate (Berger et al. 2007). This is consistent with a previous study showing that progressive deformation of the chloroplast leads to a diminished photosynthetic rate (Fig. 6c, d), which is a consequence of the systemic response to $S$. oryzae infection. K starvation decreased the systemic tolerance capacity of the host rice by enhancing the negative impacts of S. oryzae infection on leaf photosynthetic potential.

\section{Conclusions}

$S$. oryzae infection induces amino-acid accumulation in FLSs by impacting aminoacyl-tRNA biosynthesis. During the infection process, $\mathrm{K}$ starvation sharply upregulated the 1-alkyl-2-acylglycerophosphoethanolamine content but decreased the glycoglycerolipids content, which altered lipid metabolism and destroyed lipid homeostasis. $\mathrm{K}$ deficiency in rice induced $\mathrm{H}_{2} \mathrm{O}_{2}$ hyperaccumulation in diseased tissues, which caused lipid peroxidation. In contrast, a sufficient $\mathrm{K}$ supply increased antioxidant biosynthesis and activated SOD and POD enzyme activities, which alleviated the oxidative stress caused by $S$. oryzae infection. $K$ deficiency decreased the photosynthetic potential of the flag leaf by altering the morphology of chloroplasts during $S$. oryzae infection. Overall, these results emphasize the important role of $\mathrm{K}$ in alleviating $S$. oryzae infection by regulating amino acid and lipid metabolism.

\section{Materials and Methods}

\section{Plant Materials and Growth Conditions}

Rice seeds (O. sativa cv. Shengliangyou 5814, Indica rice) were disinfected with $0.5 \% \mathrm{NaClO}$ for $10 \mathrm{~h}$, followed by germination in deionized water at $30{ }^{\circ} \mathrm{C}$ for 3 days. After germination, seeds were moved to a plastic net, which was fixed on a foam material and floated in deionized water. Seven days later, the uniform seedlings were transplanted to a container $(6 \mathrm{~L})$ for 8 days $(50 \%$ strength nutrient solution for 3 days and $100 \%$ strength nutrient solution for 5 days). Finally, 15-day old seedlings were used for a pot experiment. The soil material used for the pot experiment was collected from a long-term $\mathrm{K}$ fertilizer field experiment in Wuxue County $\left(30^{\circ} 06^{\prime} 46^{\prime \prime}\right.$ N, $115^{\circ} 36^{\prime} 9^{\prime \prime}$ E), Hubei Province, central China. The soil chemical properties were as follows: total nitrogen, $1.8 \mathrm{~g} \mathrm{~kg}^{-1}$; Olsen-phosphorus (P), $13.0 \mathrm{mg} \mathrm{kg}{ }^{-1}$; available $\mathrm{K}, 30.0 \mathrm{mg} \mathrm{kg}^{-1}$; slowly available $\mathrm{K}, 288 \mathrm{mg} \mathrm{kg}^{-1}$; and organic matter, $34.0 \mathrm{~g} \mathrm{~kg}^{-1}$. The soil type was sandy loam soil with a pH of 5.60 (1:2.5, soil: deionized water), which was categorized as K-deficient soil (Zou et al. 2011). The pot experiment was conducted in a greenhouse with day/ night temperatures of $28 / 22{ }^{\circ} \mathrm{C}$, a relative humidity of $45-55 \%$, and a photon flux density of $800 \mu \mathrm{mol} \mathrm{m} \mathrm{m}^{-2} \mathrm{~s}^{-1}$.

\section{Experimental Design and S. oryzae Inoculation}

Two K levels were used in the pot experiment, which was performed with 12 replicates. In total 24 pots were used. The treatments used were (1) sufficient K supply $(+K)$, with an application rate of $1.5 \mathrm{~g} \mathrm{~K}_{2} \mathrm{O} / \mathrm{kg}$ soil (in the form of potassium sulfate), and (2) zero $K$ application (-K), with no $\mathrm{K}$ fertilizer applied to the pot. To ensure that other nutrients were not limiting factors for rice growth, $2.0 \mathrm{~g} \mathrm{~N} / \mathrm{kg}$ soil (in the form of urea) and $1.5 \mathrm{~g} \mathrm{P}_{2} \mathrm{O}_{5} / \mathrm{kg}$ soil (in the form of calcium superphosphate) were applied as basal fertilizers 1 day before transplanting. All fertilizers were mixed with the soil (total of $10 \mathrm{~kg}$ soil/pot) before watering. Three single seedlings were transplanted into each pot.

Pathogen material (S. oryzae) was isolated from diseased plants in the field, as described in our previous study (Zhang et al. 2019a). Sixty-day-old plants (at the booting stage) were used for pathogen inoculation. First, S. oryzae was incubated on potato dextrose agar medium, which was placed in an incubator at $28{ }^{\circ} \mathrm{C}$ for 8 days. Immediately afterward, sterile water was added to the plate-grown mycelium to leach a conidial suspension. For inoculation, uniform FLSs in the $-\mathrm{K}$ and $+\mathrm{K}$ treatments were selected for inoculation with $S$. oryzae at a concentration of $1 \times 10^{7}$ conidia $\mathrm{mL}^{-1}$. For each treatment, six pots were selected for inoculation. Briefly, a punch was used to make a small hole in the leaf sheath at the same position for each leaf sheath, and then 10 $\mu \mathrm{L}$ of suspended spores was injected. The control plants were injected with sterile water $(10 \mu \mathrm{L})$ as mock inoculation. Next, all pots were placed in darkness with $90 \%$ humidity for $12 \mathrm{~h}$. After successful infection, the following treatments were prepared: (1) K-starved plants with mock inoculation $(-\mathrm{K})$, (2) K-sufficient plants with mock inoculation $(+\mathrm{K})$, (3) K-starved plants with $S$. oryzae 
inoculation $(-\mathrm{K}+\mathrm{I})$, and (4) K-sufficient plants with $S$. oryzae inoculation $(+\mathrm{K}+\mathrm{I})$.

\section{Metabolome Profiling and Data Analysis}

Twelve biological replicates of fresh FLS tissues (five representative FLSs were mixed as one sample) were sampled before inoculation $(-\mathrm{K}$ and $+\mathrm{K})$ and at 5 days after inoculation $(-\mathrm{K},+\mathrm{K},-\mathrm{K}+\mathrm{I},+\mathrm{K}+\mathrm{I})$. All samples were frozen in liquid nitrogen, and then stored at $-80^{\circ} \mathrm{C}$ for extraction of metabolites, lipids, and RNA. Metabolite extraction followed the method described by with some modifications (Kumar et al. 2015). Briefly, $50 \mathrm{mg}$ of fresh sample was extracted with $1000 \mu \mathrm{L}$ of solvent (acetonitrile: methanol: water, 2:2:1, containing $1 \mu \mathrm{g} / \mathrm{mL}$ of internal standard). This process was repeated three times. Then, incubation at $-20^{\circ} \mathrm{C}$ for $1 \mathrm{~h}$ and centrifugation at $4{ }^{\circ} \mathrm{C}$ and $12,000 \mathrm{rpm}$ for $15 \mathrm{~min}$ were performed. The supernatant was stored in vial at $-80{ }^{\circ} \mathrm{C}$ until further use. Liquid chromatography with tandem mass spectrometry (LC-MS/MS) analyses were performed using an ultra-high performance liquid chromatography system (1290, Agilent Technologies, Santa Clara, CA, USA) with a UPLC HSS T3 column (2.1 mm $\times 100 \mathrm{~mm}, 1.8 \mu \mathrm{m}$, Waters, Tokyo, Japan) coupled to a $Q$ Exactive system. An accurate quadrupole time-offlight mass spectrometer (AB Triple TOF 6600, AB Sciex, Framingham, MA, USA) was used for all mass spectrometry measurements. Metabolites were identified by comparing the ion features and retention times to the reference chemical library (DeHaven et al. 2010). Student's t-test was used to determine the significance of differences in each metabolite among the $S$. oryzae-inoculated and mock-inoculated samples. The KEGG (http://www.kegg.com) database was used for annotation of the metabolites (Additional file 2: Metabolites data). Additionally, classification of metabolites was performed using the method suggested by a previous study (McLoughlin et al. 2018).

\section{Transcriptome Profiling}

Fresh samples were collected before inoculation and at 5 days after inoculation. Total RNA extraction was performed using TRIzol reagent (Invitrogen, Carlsbad, CA, USA) according to the manufacturer's instructions, followed by polyA selection for mRNA enrichment. RNA sequencing was performed by Majorbio Bio-pharm Technology Co. (Shanghai, China) using the Illumina Hiseq2500 sequencing system (San Diego, CA, USA). The sequence data were mapped to the Oryza sativa Indica Group reference genome (ASM4651v1) (Additional file 1: Table S1) from the Ensembl Plants database (http://plants. ensembl.org/Oryza_indica/Info/Index) using TopHat2 software (Kim et al. 2013). The DEGs were identified using edgeR software (version 3.24.3). We calculated the gene expression level using fragments per kilobases per million reads (FPKM, Additional file 3: FPKM values) (Mortazavi et al. 2008). To identify the DEGs between the controls and treatments, we set a false discovery rate $(\mathrm{FDR}) \leq 0.05$, absolute $\log _{2}$ (fold change $\left.(\mathrm{FC})\right) \geq 1.5$, and $p$-value $\leq 0.01$ as the discriminant standards. KOBAS2.0 was used to analyze the KEGG pathway enrichment of the DEGs (Xie et al. 2011).

\section{lonome Profiling}

Samples used for measurements of elemental contents were collected at $0,2,5$, and 10 days after $S$. oryzae inoculation. Sixteen elements were measured via inductively coupled plasma mass spectrometry (ICP-MS) using the method suggested by a previous study (McLoughlin et al. 2018). Briefly, $0.1 \mathrm{~g}$ of dry sample was digested in a mixture of $\mathrm{HNO}_{3}$ and $\mathrm{HClO}_{4}\left(4 \mathrm{HNO}_{3}: 1 \mathrm{HClO}_{4}\right)$, and the digested solution was then dissolved in $50 \mathrm{ml}$ of ultrapure water. After filtering with double filter paper, this solution was used for ICP-MS profiling. A multi-element standard (Ultra Scientific, Providence, RI, USA) was used for calibration curves to monitor the run-to-run variation.

\section{MDA, $\mathrm{H}_{2} \mathrm{O}_{2}$, Superoxide Dismutase, Peroxidase, and Lipid Extraction Assays}

MDA, $\mathrm{H}_{2} \mathrm{O}_{2}$ content, and SOD and POD enzyme activities were measured according to the method described by Djanaguiraman et al (2009). Fresh FLSs sampled before and at 5 days after $S$. oryzae inoculation were used for lipid extraction. The lipid content measurements were performed using electrospray ionization tandem mass spectrometry (ESI-MS/MS) according to the method suggested by a previous study with some modifications (Djanaguiraman et al. 2009; Liu et al. 2015). Briefly, to inactivate phospholipase activity, samples were placed in tubes containing $3.0 \mathrm{ml}$ isopropanol with $0.01 \%$ butylated hydroxytoluene (BHT) for $20 \mathrm{~min}$, followed by extraction with $1.5 \mathrm{ml}$ chloroform and $0.5 \mathrm{ml}$ water for $1.5 \mathrm{~h}$. Then, the samples were extracted with $4.0 \mathrm{ml}$ chloroform/methanol $(2: 1, \mathrm{v} / \mathrm{v})$ containing $0.01 \%$ BHT. This extraction step was repeated six times. Finally, the lipid extracts were washed with $1 \mathrm{mM} \mathrm{KCl}$ and sterile water. Nitrogen gas was used to dry the lipid phases. Then, the extracts were dissolved in chloroform and used for ESI-MS/MS analysis. The remaining FLS tissues were dried in an oven, and the lipid content was calculated based on the dry weight. Automated ESI-MS/MS was used for lipid analysis. The approach and data processing followed a previously described method, and internal standards were used for the calculation of lipid contents (Narayanan et al. 2016). 
MGDG, DGDG, PC and PG contents were measured independently with six biological replicates.

\section{Chlorophyll Fluorescence Imaging, Photosynthetic Rate, and Chloroplast Ultrastructure}

Chlorophyll fluorescence imaging was performed using the MINI-Version Imaging-PAM (IMAG-MIN/B, Walz, Effeltrich, Germany) system following the method suggested by Lu et al. (2017). At 5 days upon inoculation, the flag leaf was selected for gas-exchange measurements, which were performed using a portable photosynthesis system (Li-6400XT, Li-Cor, Inc., Lincoln, NE, USA) from 9:00 to 11:30 a.m. Leaves used for light and $\mathrm{CO}_{2}$ responsecurve measurements were previously acclimated to saturating light for $30 \mathrm{~min}$. Thirteen levels of photosynthetic photon flux density (PPFD) $(2000,1500,1200,1000$, $800,600,400,200,150,100,50,25$, and $0 \mu \mathrm{mol} \mathrm{m}^{-2} \mathrm{~s}^{-1}$ ) were used to determine the light-response curve. Additionally, a series of $\mathrm{CO}_{2}$ concentrations $(400,300,200$, $100,50,400,600,800,1000,1200$, and $1500 \mu \mathrm{mol} \mathrm{CO}$ $\mathrm{mol}^{-1}$ ) was used to determine the $\mathrm{CO}_{2}$ response curve at a constant PPFD of $1500 \mu \mathrm{mol} \mathrm{m}^{-2} \mathrm{~s}^{-1}$. After the curve measurements, flag leaf and FLS segments were sampled for ultrastructural observations following the method described by Lu et al. (2016).

\section{Statistical Analysis}

Data analysis was performed using the least significant difference test with $\alpha=0.05$ in SPSS 19.0 (SPSS, Inc., Chicago, IL, USA). Figures were drawn using Origin 9.0 software (OriginLab Corporation, Northampton, MA, USA) and R3.6.1 (R Foundation for Statistical Computing, Vienna, Austria) with the "pheatmap" package.

\section{Supplementary Information}

The online version contains supplementary material available at https://doi. org/10.1186/s12284-021-00524-6.

Additional file 1: Fig. S1 GO function classification of DEGs. Fig. S2.

Trypan blue staining of FLSs. Fig. S3 K deficiency alters the elemental contents of FLS during S. oryzae infection. Fig. $\mathbf{S 4} \mathrm{K}$ concentrations of healthy and diseased plants among different organs. Table S1 Mapping statistics of K-starved rice and K-sufficient rice at 0 and 5 days post inoculation. Table S2 Expression profile of lipid metabolism related genes in the FLS. Table S3 Lipid species related to phospholipids metabolism based on the top 10 VIP scores. Table S4 Morphological parameters of chloroplasts. Table S5 Photosynthetic characteristics of flag leaf.

Additional file 2. Metabolites data.

Additional file 3. FPKM values.

Acknowledgements

We thank Dr. Wang Yong College of Plant Science \& Technology, Huazhong Agricultural University, China, for his excellent support in screening and identification of the pathogen material.

\section{Authors' Contributions}

The experiment was designed by T.R., R.C., J.L. and X.L., J.Z. performed the experiment and write the original draft. Z.L. helped revise the article. All authors read and approved the final manuscript.

\section{Funding}

This work was financially supported by National Natural Science Foundation of China (31872174) and the Fundamental Research Funds for the Central Universities (2662018YJ026).

\section{Availability of Data and Materials}

The datasets used and/or analysed during the current study are available from the corresponding author on reasonable request.

\section{Declarations}

Ethics Approval and Consent to Participate

Not applicable.

\section{Consent for Publication}

Not applicable.

\section{Competing Interests}

The authors declare that they have no competing interests.

\section{Author details}

${ }^{1}$ Key Laboratory of Arable Land Conservation (Middle and Lower Reaches of Yangtze River), Ministry of Agriculture and Rural Affairs, People's Republic of China, Wuhan 430070, China. ${ }^{2}$ Microelement Research Center, Huazhong Agricultural University, Wuhan 430070, China.

Received: 28 June 2021 Accepted: 9 September 2021

Published online: 17 September 2021

\section{References}

Adigun OA, Nadeem M, Pham TH, Jewell LE, Cheema M, Thomas R (2021) Recent advances in biochemical, molecular and physiological aspects of membrane lipid derivatives in plant pathology. Plant Cell Environ 44:1-16

Amtmann A, Troufflard S, Armengaud P (2008) The effect of potassium nutrition on pest and disease resistance in plants. Physiol Plant 133:682-691

Armengaud P, Breitling R, Amtmann A (2010) Coronatine-insensitive 1 (COI1) mediates transcriptional responses of Arabidopsis thaliana to external potassium supply. Mol Plant 3:390-405

Berger S, Benediktyová Z, Matouš K, Bonfig K, Mueller MJ, Nedbal L, Roitsch T (2007) Visualization of dynamics of plant-pathogen interaction by novel combination of chlorophyll fluorescence imaging and statistical analysis: differential effects of virulent and avirulent strains of $P$. syringae and of oxylipins on A. thaliana. J Exp Bot 58:797-806

Bigirimana VDP, Hua GK, Nyamangyoku OI, Höfte M (2015) Rice sheath rot: an emerging ubiquitous destructive disease complex. Front Plant Sci 6:1066

Chen LQ (2014) SWEET sugar transporters for phloem transport and pathogen nutrition. New Phytol 201:1150-1155

Conconi A, Miquel M, Ryan CA (1996) Intracellular levels of free linolenic and linoleic acids increase in tomato leaves in response to wounding. Plant Physiol 111:797-803

DeHaven CD, Evans AM, Dai H, Lawton KA (2010) Organization of GC/MS and LC/MS metabolomics data into chemical libraries. J Cheminform 2:9

Demidchik V, Straltsova D, Medvedev SS, Pozhvanov GA, Sokolik A, Yurin V (2014) Stress-induced electrolyte leakage: the role of $\mathrm{K}^{+}$-permeable channels and involvement in programmed cell death and metabolic adjustment. J Exp Bot 65:1259-1270

Djanaguiraman M, Annie Sheeba J, Durga Devi D, Bangarusamy U (2009) Cotton leaf senescence can be delayed by nitrophenolate spray through enhanced antioxidant defence system. J Agron Crop Sci 195:213-224

Duchêne AM, Giritch A, Hoffmann B, Cognat V, Lancelin D, Peeters NM, Small ID (2005) Dual targeting is the rule for organellar aminoacyl-tRNA synthetases in Arabidopsis thaliana. Proc Natl Acad Sci USA 102:16484-16489 
Fagard M, Launay A, Clément G, Courtial J, Dellagi A, Farjad M, MasclauxDaubresse C (2014) Nitrogen metabolism meets phytopathology. J Exp Bot 65:5643-5656

Gao M, Yin X, Yang W, Lam SM, Tong X, Liu J, He Z (2017) GDSL lipases modulate immunity through lipid homeostasis in rice. PLoS Pathog 13:e1006724

Hittalmani S, Mahesh HB, Mahadevaiah C, De Prasannakumar MK (2016) novo genome assembly and annotation of rice sheath rot fungus Sarocladium oryzae reveals genes involved in Helvolic acid and Cerulenin biosynthesis pathways. BMC Genomics 17:271

Hu W, Lv X, Yang J, Chen B, Zhao W, Meng Y, Zhou Z, Oosterhuis DM (2016) Effects of potassium deficiency on antioxidant metabolism related to leaf senescence in cotton (Gossypium hirsutum L.). Field Crop Res 191:139-149

Kim D, Pertea G, Trapnell C, Pimentel H, Kelley R, Salzberg SL (2013) TopHat2: accurate alignment of transcriptomes in the presence of insertions, deletions and gene fusions. Genome Biol 14:R36

Kumar Y, Dholakia BB, Panigrahi P, Kadoo NY, Giri AP, Gupta VS (2015) Metabolic profiling of chickpea-Fusarium interaction identifies differential modulation of disease resistance pathways. Phytochemistry 116:120-129

Kunz HH, Gierth M, Herdean A, Satoh-Cruz M, Kramer DM, Spetea C, Schroeder J (2014) Plastidial transporters KEA1, -2, and-3 are essential for chloroplast osmoregulation, integrity, and $\mathrm{pH}$ regulation in Arabidopsis. Proc Natl Acad Sci USA 111:7480-7485

Liu Y, Wang G, Wang X (2015) Role of aminoalcoholphosphotransferases 1 and 2 in phospholipid homeostasis in Arabidopsis. Plant Cell 27:1512-1528

Lu Z, Lu J, Pan Y, Lu P, Li X, Cong R, Ren T (2016) Anatomical variation of mesophyll conductance under potassium deficiency has a vital role in determining leaf photosynthesis. Plant Cell Environ 39:2428-2439

Lu Z, Pan Y, Hu W, Cong R, Ren T, Guo S, Lu J (2017) The photosynthetic and structural differences between leaves and siliques of Brassica napus exposed to potassium deficiency. BMC Plant Biol 17:240

Marschner H (2012) Marschner's mineral nutrition of higher plants, 3rd edn. Academic Press, London, pp 178-189

McLoughlin F, Augustine RC, Marshall RS, Li F, Kirkpatrick LD, Otegui MS, Vierstra RD (2018) Maize multi-omics reveal roles for autophagic recycling in proteome remodelling and lipid turnover. Nat Plants 4:1056-1070

Mortazavi A, Williams BA, McCue K, Schaeffer L, Wold B (2008) Mapping and quantifying mammalian transcriptomes by RNA-Seq. Nat Methods 5:621-628

Narayanan S, Tamura PJ, Roth MR, Prasad PV, Welti R (2016) Wheat leaf lipids during heat stress: I. High day and night temperatures result in major lipid alterations. Plant Cell Environ 39:787-803

Nianiou-Obeidat I, Madesis P, Kissoudis C, Voulgari G, Chronopoulou E, Tsaftaris A, Labrou NE (2017) Plant glutathione transferase-mediated stress tolerance: functions and biotechnological applications. Plant Cell Rep 36:791-805

Nishizawa A, Yabuta Y, Shigeoka S (2008) Galactinol and raffinose constitute a novel function to protect plants from oxidative damage. Plant Physiol 147:1251-1263

Nita M, Grzybowski A (2016) The role of the reactive oxygen species and oxidative stress in the pathomechanism of the age-related ocular diseases and other pathologies of the anterior and posterior eye segments in adults. Oxid Med Cell Longev 2016:3164734

Peeters KJ, Ameye M, Demeestere K, Audenaert K, Höfte M (2020) Auxin, abscisic acid and jasmonate are the central players in rice sheath rot caused by Sarocladium oryzae and Pseudomonas fuscovaginae. Rice 13(1):1-16

Perlikowski D, Kierszniowska S, Sawikowska A, Krajewski P, Rapacz M, Eckhardt $\ddot{A}$, Kosmala A (2016) Remodeling of leaf cellular glycerolipid composition under drought and re-hydration conditions in grasses from the LoliumFestuca complex. Front Plant Sci 7:1027

Raffaele S, Leger A, Roby D (2009) Very long chain fatty acid and lipid signaling in the response of plants to pathogens. Plant Signal Behav 4:94-99

Robison FM, Turner MF, Jahn CE, Schwartz HF, Prenni JE, Brick MA, Heuberger AL (2018) Common bean varieties demonstrate differential physiological and metabolic responses to the pathogenic fungus Sclerotinia sclerotiorum. Plant Cell Environ 41:2141-2154

Rojas CM, Senthil-Kumar M, Tzin V, Mysore K (2014) Regulation of primary plant metabolism during plant-pathogen interactions and its contribution to plant defense. Front Plant Sci 5:17

Ruan J, Zhou Y, Zhou M, Yan J, Khurshid M, Weng W, Zhang K (2019) Jasmonic acid signaling pathway in plants. Int J Mol Sci 20:2479

Sakthivel N (2001) Sheath rot disease of rice: current status and control strategies. In: Major fungal diseases of rice, pp 271-283. Springer, Dordrecht

Shannon P, Markiel A, Ozier O, Baliga NS, Wang JT, Ramage D, Amin N, Schwikowski B, Ideker T (2003) Cytoscape: a software environment for integrated models of biomolecular interaction networks. Genome Res 13:2498-2504

Sheppard K, Yuan J, Hohn MJ, Jester B, Devine KM, Söll D (2008) From one amino acid to another: tRNA-dependent amino acid biosynthesis. Nucleic Acids Res 36:1813-1825

Shi X, Long Y, He F, Zhang C, Wang R, Zhang T, Wu W, Hao Z, Wang Y, Wang G, Ning Y (2018) The fungal pathogen Magnaporthe oryzae suppresses innate immunity by modulating a host potassium channel. PLoS Pathog 14:e1006878

Shin R, Schachtman DP (2004) Hydrogen peroxide mediates plant root cell response to nutrient deprivation. Proc Natl Acad Sci USA 101:8827-8832

Torres MA, Jones JD, Dangl JL (2005) Pathogen-induced, NADPH oxidasederived reactive oxygen intermediates suppress spread of cell death in Arabidopsis thaliana. Nat Genet 37:1130-1134

Torres-Franklin ML, Repellin A, Huynh VB, d'Arcy-Lameta A, Zuily-Fodil Y, PhamThi AT (2009) Omega-3 fatty acid desaturase (FAD3, FAD7, FAD8) gene expression and linolenic acid content in cowpea leaves submitted to drought and after rehydration. Environ Exp Bot 65:162-169

Triantaphylidès C, Krischke M, Hoeberichts FA, Ksas B, Gresser G, Havaux M, Breusegem FV, Mueller MJ (2008) Singlet oxygen is the major reactive oxygen species involved in photooxidative damage to plants. Plant Physiol 148:960-968

Troufflard S, Mullen W, Larson TR, Graham IA, Crozier A, Amtmann A, Armengaud $P$ (2010) Potassium deficiency induces the biosynthesis of oxylipins and glucosinolates in Arabidopsis thaliana. BMC Plant Biol 10:172

Xie C, Mao X, Huang J, Ding Y, Wu J, Dong S, Kong L, Gao G, Li C-Y, Wei L (2011) KOBAS 2.0: a web server for annotation and identification of enriched pathways and diseases. Nucleic Acids Res 39:316-322

Zhang J, Lu Z, Pan Y, Ren T, Cong R, Lu J, Li X (2019a) Potassium deficiency aggravates yield loss in rice by restricting the translocation of nonstructural carbohydrates under Sarocladium oryzae infection condition. Physiol Plant 167:352-364

Zhang X, Xu Y, Huang B (2019b) Lipidomic reprogramming associated with drought stress priming-enhanced heat tolerance in tall fescue (Festuca arundinacea). Plant Cell Environ 42:947-958

Zhang J, Hou W, Ren T, Cong R, Zhao Z, Lu J, Li X (2020) Applying potassium fertilizer improves sheath rot disease tolerance and decreases grain yield loss in rice (Oryza sativa L.). Crop Prot 139:105392

Zhou L, Zhang Y, Rao L, Cao Y, Peng Y (2008) Galactolipid depletion in blast fungus-infected rice leaves. Ann Appl Biol 153:395-400

Zoeller M, Stingl N, Krischke M, Fekete A, Waller F, Berger S, Mueller MJ (2012) Lipid profiling of the Arabidopsis hypersensitive response reveals specific lipid peroxidation and fragmentation processes: biogenesis of pimelic and azelaic acid. Plant Physiol 160:365-378

Zou J, Lu J, Li Y, Li X (2011) Regional evaluation of winter rapeseed response to $K$ fertilization, K use efficiency, and critical level of soil $K$ in the Yangtze River Valley. J Integr Agric 10:911-920 ((in Chinese))

\section{Publisher's Note}

Springer Nature remains neutral with regard to jurisdictional claims in published maps and institutional affiliations. 